\title{
New Integrable Problem of Classical Mechanics
}

\author{
O. I. Bogoyavlensky \\ V. A. Steklov Mathematical Institute, The Academy of Sciences of the USSR, \\ SU-117333 Moscow, USSR
}

\begin{abstract}
Complete integrability in Liouville's sense is proven for rotation of an arbitrary rigid body with a fixed point in a Newtonian field with an arbitrary homogeneous quadratic potential. A consequence is the complete integrability of rotation of a rigid body with fixed center of mass in the field of arbitrary sufficiently remote objects (in the second approximation). Explicit formulae are obtained expressing angular velocities of the rigid body in terms of $\theta$-functions for Riemannian surfaces. Integrable cases are found for rotation of a rigid body in nonlinear Newtonian potential fields.
\end{abstract}

\section{Introduction and Summary}

Investigation of dynamics of a rigid body with a fixed point in a Newtonian potential field $\varphi\left(x^{1}, x^{2}, x^{3}\right)$ is a classical problem of mechanics. For a linear potential, $\varphi=a_{1} x^{1}+a_{2} x^{2}+a_{3} x^{3}$, rotation of a rigid body is described by the Euler-Poisson equations, that are not integrable in the general case. The dynamics is integrable only in three special cases which were discovered by Euler [1], Lagrange [2], and Kowalewski [3]. In the two former cases the problem is integrated in terms of elliptic functions, in the Kowalewski case - in terms of the Riemann $\theta$-functions of two variables.

The main result of the present work is that rotation of an arbitrary rigid body with a fixed point in a Newtonian field given by an arbitrary quadratic potential, $\varphi=\frac{1}{2} \sum_{i, j=1}^{3} a_{i j} x^{i} x^{j}$, is always completely integrable in Liouville's sense. The dynamical equations are integrated explicitly in terms of the Riemann $\theta$-functions of 4 variables, restricted to a three-dimensional manifold (a Prym variety).

The problem of rotation of a rigid body in a quadratic potential field appears naturally in the following situation. Let us consider an arbitrary rigid body $T$ fixed at its center of mass $O$ under the action of the gravitational field of an object $V$ (consisting, say, of several disconnected massive bodies). Suppose $l$ is a linear size of the body $T, R$ is the minimal distance from the point $O$ to the field source $V$, and 
$l \ll R$. If the center of mass $O$ is fixed, the linear terms in the expansion of the Newtonian potential $\varphi$ near the point $O$ produce no effect on rotation of the body $T$, and quadratic terms are dominating for $l \ll R$. Therefore, Theorems 1 and 2 , which are crucial in the present work, lead to the following statement: in the Newtonian field of a remote gravitating object $V$, rotation of a rigid body which is fixed at its center of mass is a system completely integrable in Liouville's sense in the second-order approximation; the problem is integrated explicitly in terms of Riemann's $\theta$-functions.

Until now the only integrable case known for this problem (which was investigated earlier in [4-6]) was the case, where the gravitational potential $\varphi$ had an axial symmetry (with the center $O$ in the symmetry axis). In that case the potential was of the form $\varphi=a\left(x^{1}\right)^{2}$ (Brun's problem), and the equations describing the rotation of a rigid body $T$ were reduced there to Clebsch's case of integrability [7] for the Kirchhoff equations.

In Sect. 2 of the present work we show that the equations describing rotation of a rigid body in a quadratic potential field are Euler's equations in a space conjugate to a nine-dimensional Lie algebra $L_{9}$, so these are the Hamiltonian equations in six-dimensional orbits $\mathscr{M}^{6}$. The resulting Hamiltonian systems in the symplectic manifolds $\mathscr{M}^{6}$ have three involutive first integrals $J_{1}, J_{2}, J_{3}$ (the integral $J_{1}$ is the system Hamiltonian), so they are completely integrable in Liouville's sense. Note that the dynamics of trajectories in the problem in view is a linear winding of the three-dimensional invariant tori $\mathbb{T}^{3}$. For all the classical integrable cases [1-4, 7-11], which are known for real (three-dimensional) physical problems, dynamics of the trajectories takes place in two-dimensional tori $\mathbb{T}^{2}$, so the authors used the method of the "last Jacobi factor" to prove the integrability. In the problem under consideration the manifolds, where all the first integrals of the system acquire fixed values, are three-dimensional (as in the general case of the Euler-Poisson equations), so the method of the last Jacobi factor is not applicable here, and the proof of the system's integrability is based upon an investigation of its Hamiltonian structure.

In Sect. 3 we present an equivalent representation of the dynamical system in terms of the isospectral deformation equations, depending on a spectral parameter $E$ (some dynamical systems with a spectral parameter were studied previously [12-19]). In view of the existence of such a representation, the system is related naturally with a family of Riemannian surfaces $\Gamma$ which depend on magnitudes of the first integrals and have the genus $g(\Gamma)=4$. The arising Riemannian surfaces are not hyperelliptic, this is an essential feature in which the physical problem in view is different from all known classical integrable cases [1-4, 7-11]. The Riemannian surfaces $\Gamma$ admit an involution $\sigma: \Gamma \rightarrow \Gamma, \sigma^{2}=\mathrm{id}$. It is shown that the dynamics of trajectories of the system is linearized in the three-dimensional Prym variety, $\operatorname{Prym}_{\sigma} \Gamma \subset \mathrm{Jac} \Gamma$ (the Jacobi variety $\mathrm{Jac} \Gamma$ is four-dimensional). Explicit formulae have been obtained which express the angular rotation velocities of the rigid body $\omega_{i}^{j}(t)$ in terms of the $\theta$-functions of the Riemannian surfaces $\Gamma$.

In Sect. 4 the integrability in terms of the Riemann $\theta$-functions is proven for an $n$-dimensional analog of the rotation problem for a rigid body in a quadratic potential field, and it is shown that the dynamic is linearized in the corresponding Prym varieties. In Sect. 5 we indicate certain integrable cases for the rotation problem for a rigid body in some more complicated Newtonian fields. 


\section{Liouville Integrability for Rotation of an Arbitrary Rigid Body with a Fixed Point in Newtonian Field with an Arbitrary Quadratic Potential}

Equations describing rotation of a rigid body $T$ with a fixed point $O$ are considered in a reference frame which is related to the body. Let $\varphi\left(x^{1}, x^{2}, x^{3}\right)$ be the Newtonian potential in the resting frame $F$, having its center at the point $O ; \boldsymbol{\alpha}, \boldsymbol{\beta}, \boldsymbol{\gamma}$ are the unit basis vectors of the resting reference frame, as given in the moving frame $S$. The potential function is defined as

$$
U(\boldsymbol{\alpha}, \boldsymbol{\beta}, \boldsymbol{\gamma})=\int_{T} \varrho(\mathbf{r}) \varphi((\mathbf{r}, \boldsymbol{\alpha}),(\mathbf{r}, \boldsymbol{\beta}),(\mathbf{r}, \boldsymbol{\gamma})) d r^{1} d r^{2} d r^{3},
$$

where $\varrho(r)$ is the mass density of the rigid body $T$ at a point $r$. The equations describing rotation of the rigid body $T$ in the Newtonian potential field $\varphi\left(x^{1}, x^{2}, x^{3}\right)$, in the reference frame $S$, are as follows

$$
\begin{aligned}
\dot{\mathbf{M}} & =\mathbf{M} \times \boldsymbol{\omega}+(\partial U / \partial \boldsymbol{\alpha}) \times \boldsymbol{\alpha}+(\partial U / \partial \boldsymbol{\beta}) \times \boldsymbol{\beta}+(\partial U / \partial \gamma) \times \boldsymbol{\gamma}, \\
\dot{\boldsymbol{\alpha}} & =\boldsymbol{\alpha} \times \boldsymbol{\omega}, \quad \dot{\boldsymbol{\beta}}=\boldsymbol{\beta} \times \boldsymbol{\omega}, \quad \dot{\gamma}=\boldsymbol{\gamma} \times \boldsymbol{\omega},
\end{aligned}
$$

where $\mathbf{M}$ is the angular momentum vector, and $\boldsymbol{\omega}$ is the angular velocity vector. Their components are related by the equality

$$
M_{i}=\sum_{k=1}^{3} I_{i k} \omega_{k}, \quad I_{i k}=\int_{T} \varrho(\mathbf{r})\left(\delta_{i k} \sum_{\ell=1}^{3}\left(r^{\ell}\right)^{2}-r^{i} r^{k}\right) d r^{1} d r^{2} d r^{3},
$$

where $I_{i k}$ are components of the inertia tensor of the rigid body $T$ in the reference frame $S$.

Theorem 1. Rotation of an arbitrary rigid body $T$ with a fixed point $O\left(x^{1}=x^{2}\right.$ $=x^{3}=0$ ) in a Newtonian field given by an arbitrary quadratic potential

$$
\varphi(\mathbf{x})=2^{-1} \sum_{i, j=1}^{3} a_{i j} x^{i} x^{j}
$$

is completely integrable in Liouville's sense.

Note that in Theorem 1 we mean an arbitrary point $O$ (not necessarily the center of mass of the body), and the potential $\varphi(\mathbf{x})$ may not satisfy the Laplace equation, $\Delta \varphi=0$.

We introduce a basis in the resting frame $F$ with the unit vectors along the principal axes of the quadratic form in (2.4); then we have $2 \varphi(\mathbf{x})=a_{1}\left(x^{1}\right)^{2}+a_{2}\left(x^{2}\right)^{2}$ $+a_{3}\left(x^{3}\right)^{2}$. Let the basis vectors of the reference frame $S$ coincide with the principal axes of the inertia tensor in Eq. (2.3), so $I_{i k}=I_{i} \delta_{i k}$. Now the potential function in Eq. (2.1) is

$$
\begin{aligned}
2 U= & U_{0}-a_{1}\left(I_{1} \alpha_{1}^{2}+I_{2} \alpha_{2}^{2}+I_{3} \alpha_{3}^{2}\right)-a_{2}\left(I_{1} \beta_{1}^{2}+I_{2} \beta_{2}^{2}+I_{3} \beta_{3}^{2}\right) \\
& -a_{3}\left(I_{1} \gamma_{1}^{2}+I_{2} \gamma_{2}^{2}+I_{3} \gamma_{3}^{2}\right), \\
U_{0}= & \left(a_{1}+a_{2}+a_{3}\right)\left(I_{1}+I_{2}+I_{3}\right) / 2 .
\end{aligned}
$$

Use the known isomorphism between three-dimensional vectors, with components $v^{i}$, and skew-symmetric $3 \times 3$ matrices, with elements $V_{j k}$,

$$
v^{i} \rightarrow V_{j k}=-\sum_{i=1}^{3} v^{i} \varepsilon_{i j k}
$$


This isomorphism maps the vector product $\mathbf{x} \times \mathbf{y}$ to a commutator of the matrices, $[X, Y]=X Y-Y X$. Apply this isomorphism to the vectors $\boldsymbol{\alpha}, \boldsymbol{\beta}, \boldsymbol{\gamma}, \mathbf{M}, \boldsymbol{\omega}$; in view of (2.2), the corresponding matrices $\alpha, \beta, \gamma, M, \omega$ satisfy the following equations

$$
\begin{aligned}
\dot{M} & =[M, \omega]+a_{1}[\alpha, C \alpha+\alpha C]+a_{2}[\beta, C \beta+\beta C]+a_{3}[\gamma, C \gamma+\gamma C], \\
\dot{\alpha} & =[\alpha, \omega], \quad \dot{\beta}=[\beta, \omega], \quad \dot{\gamma}=[\gamma, \omega],
\end{aligned}
$$

where the matrix $C$ has the elements $C_{i j}=\left[\frac{1}{2}\left(I_{1}+I_{2}+I_{3}\right)-I_{i}\right] \delta_{i j}$, and $M_{i j}=I_{k} \omega_{i j}$ $(i, j, k=1,2,3)$. Because of Eqs. (2.7), we have

$$
\left(\alpha^{2}\right)^{\cdot}=\left[\alpha^{2}, \omega\right], \quad\left(\beta^{2}\right)^{\cdot}=\left[\beta^{2}, \omega\right], \quad\left(\gamma^{2}\right)^{\cdot}=\left[\gamma^{2}, \omega\right] .
$$

We introduce a new matrix $u=a_{1} \alpha^{2}+a_{2} \beta^{2}+a_{3} \gamma^{2}$, and use the following identities,

$$
[x, C x+x C]=\left[x^{2}, C\right], \quad[u, C]=-[u, I] .
$$

A consequence of Eqs. (2.7) is

$$
\dot{M}=[M, \omega]-[u, I], \quad \dot{u}=[u, \omega] .
$$

Here the matrices $M$ and $\omega$ are skew-symmetric, matrices $u$ and $I$ are symmetric. Another derivation of Eqs. (2.8), in a more general case, is presented in Sect. 5.

Equations (2.8) are the Euler equations (a definition is given in [20]) in the space conjugate to a Lie algebra $L_{9}$, elements of which are $l=M+u$, where $M$ and $u$ are $3 \times 3$ matrices, $M^{t}=-M, u^{t}=u$. The commutators are defined as follows

$$
[M, u]=M u-u M, \quad\left[M_{1}, M_{2}\right]=M_{1} M_{2}-M_{2} M_{1}, \quad\left[u_{1}, u_{2}\right]=0 .
$$

The orbits $\mathscr{O}$ of action of the corresponding Lie group $G_{9}$ in $L_{9}^{*}$ are symplectic manifolds $\mathscr{M}^{6}=R^{3} \times \mathrm{SO}(3)=T(\mathrm{SO}(3))$, the latter is a tangent bundle to the Lie group $\mathrm{SO}(3)$. The manifolds $\mathscr{M}^{6}$ are determined by the conditions $\lambda_{j}(u)=$ const, where $\lambda_{j}(u)$ are eigenvalues of the matrix $u$. If $\lambda_{1}=\lambda_{2} \neq \lambda_{3}$, the orbit is $\mathscr{M}^{5}=R^{3} \times S^{2}$, and if $\lambda_{1}=\lambda_{2}=\lambda_{3}$ the orbit is just $R^{3}$. The Poisson brackets in $L_{9}^{*}$ are introduced as follows

$$
\{f, g\}=\sum_{i, j, k} C_{i j}^{k} x^{k} \frac{\partial f}{\partial x_{i}} \frac{\partial g}{\partial x_{j}},
$$

where $C_{i j}^{k}$ are the structure constants for the Lie algebra $L_{9}$ in a basis $x^{i}$ (linear functions in $L_{9}^{*}$, say $x^{i}$, belong to the Lie algebra $L_{9}$, by definition). The functions $\lambda_{j}(u)$, or equivalently, $\operatorname{Tr}(u), \operatorname{Tr}\left(u^{2}\right), \operatorname{Tr}\left(u^{3}\right)$, are annulators of the Poisson brackets (2.10); that is to say, for any function $f$ in $L_{9}^{*}$ one has $\left\{f, \lambda_{j}(u)\right\}=0$ (this fact is an analogue of that established in [21] for the Kirchhoff equations). Restrictions of the Poisson brackets to the manifold $\mathscr{M}^{6}\left[\lambda_{j}(u)=c_{j}\right]$ is non-degenerate.

Equations (2.8) have a Hamiltonian form,

$$
\dot{M}_{i j}=\left\{M_{i j}, H\right\}, \quad \dot{u}_{i j}=\left\{u_{i j}, H\right\},
$$

where the Hamiltonian is $H=J_{1}=\operatorname{Tr}\left(\frac{1}{2} M \omega-u I\right)$.

Let us introduce a matrix $B$ with components $B_{i j}=I_{1} I_{2} I_{3} I_{i}^{-1} \delta_{i j}$. It is used to write down two additional first integrals of the system (2.8),

$$
J_{2}=\operatorname{Tr}\left(\frac{1}{2} M^{2}+B u\right), \quad J_{3}=\operatorname{Tr}\left(M^{2} u+B u^{2}\right) .
$$


Evidently, the integrals $J_{1}, J_{2}, J_{3}$ are independent functions. Because of Eqs. (2.11), one has $\dot{J}_{2}=\left\{J_{2}, J_{1}\right\}=0, \dot{J}_{3}=\left\{J_{3}, J_{1}\right\}=0$. A direct calculation, using Eqs. (2.9) and (2.10) shows that the Poisson bracket of $J_{2}$ and $J_{3}$ is vanishing, so the three integrals $J_{1}, J_{2}, J_{3}$ are in involution. Therefore, the Hamiltonian system given in Eqs. (2.8) (2.11) is completely integrable in Liouville's sense in six-dimensional symplectic sub-manifolds $\mathscr{M}^{6}$, and the dynamics of trajectories is quasi-periodic in three-dimensional tori $\mathbb{T}^{3}$ in $L_{9}^{*}$, which are defined by the conditions $J_{i}=c_{i}$, $\lambda_{j}(u)=k_{j}$.

The general equations (2.2) describing rotation of a rigid body in an arbitrary Newtonian field around a fixed point are Euler equations in a space $L_{12}^{*}$ which is conjugate to the Lie algebra $L_{12}$, that has the following commutators in a basis $X_{i}$, $Y_{j}^{\alpha}(i, j, k, \alpha, \beta=1,2,3)$,

$$
\left[X_{i}, X_{j}\right]=\varepsilon_{i j k} X_{k}, \quad\left[X_{i}, Y_{j}^{\alpha}\right]=\varepsilon_{i j k} Y_{k}^{\alpha}, \quad\left[Y_{i}^{\alpha}, Y_{j}^{\beta}\right]=0 .
$$

Dynamical equations (2.2) have the energy integral, $J_{1}=\frac{1}{2}(\mathbf{M}, \boldsymbol{\omega})-U(\boldsymbol{\alpha}, \boldsymbol{\beta}, \boldsymbol{\gamma})$, and six additional geometrical integrals $J_{2}, \ldots, J_{7}$ that are constant scalar pair products of the vectors $\boldsymbol{\alpha}, \boldsymbol{\beta}, \boldsymbol{\gamma}$. The surface, where all the integrals $J_{2}, \ldots, J_{7}$ are constant is a submanifold $\mathscr{M}_{1}^{6}=T(\mathrm{SO}(3))$. Equations (2.2) are Hamiltonian in $\mathscr{M}_{1}^{6}$, with the Hamiltonian $J_{1}$, within the symplectic structure defined in Eqs. (2.10)-(2.13). These equations are the first example of physically relevant Euler equations in a Lie algebra, with an arbitrarily nonlinear potential function $U$ in the Hamiltonian $J_{1}$. [In the definition (2.1), the density $\varrho(\mathbf{r})$ is an arbitrary positive function, and the potential $\varphi\left(x^{1}, x^{2}, x^{3}\right)$ satisfies the single condition $\Delta \varphi=0$.] In all other known examples, the Hamiltonian $H$ is a quadratic function.

With the potential function of type (2.5), the Euler equations (2.2) are reduced to Euler equations (2.8); in this case the manifolds $\mathscr{M}_{1}^{6}$ and $\mathscr{M}^{6}$ are identical. The symplectic structures in these manifolds, which are introduced by non-isomorphic systems of commutators, (2.13) and (2.9), are apparently different, though the Hamiltonian functions of both systems are coinciding.

\section{Integration of the Dynamics in Terms of Riemann's $\theta$-Functions}

1. There is an equivalent representation of Eqs. (2.8) as isospectral deformation equations depending on an arbitrary parameter $E$,

$$
\dot{L}=[L, Q], \quad L=B E^{2}+M E+u, \quad Q=\omega-E I .
$$

Actually, the dynamical equation in (3.1) is a third-order polynomial in $E$. The requirement that the coefficients at $E^{k}$ must all vanish results in the following equations,

$$
\begin{gathered}
E^{3}:[B, I]=0, \quad E^{2}:[B, \omega]-[M, I]=0, \\
E^{1}: \dot{M}=[M, \omega]-[u, I], \quad E^{0}: \dot{u}=[u, \omega] .
\end{gathered}
$$

The first and the second equations in (3.2) hold because of the definition of the matrix $B$ and as $M_{i j}=I_{k} \omega_{i j}(i, j, k=1,2,3)$; the third and the fourth equations in (3.2) coincide with the system (2.8). 
The integrals (2.12) are the coefficients at $E^{2}$ in the polynomial decompositions of the functions $\operatorname{Tr}\left(L^{2}(E)\right)$ and $\operatorname{Tr}\left(L^{3}(E)\right)$, and they are independent of $t$ in view of Eq. (3.1).

The eigenvalues of the matrix $L, w_{1}, w_{2}, w_{3}$ are integrals of the system (3.1); they satisfy the equation

$$
R(w, E)=\operatorname{det}\left(B E^{2}+M E+u-w \cdot 1\right)=0 .
$$

Thus a Riemannian surface $\Gamma$, determined by Eq. (3.3), is related naturally to Eq. (3.1). All coefficients in the polynomial present in (3.3) are first integrals of the system (2.8), and they are expressed in terms of the following six first integrals

$$
\begin{gathered}
J_{1}=\operatorname{Tr}\left[\frac{1}{2}(M \omega-u I)\right], \quad J_{2}=\operatorname{Tr}\left(\frac{1}{2} M^{2}+B u\right), \\
J_{3}=\operatorname{Tr}\left(M^{2} u+B u^{2}\right), \quad J_{4}=\operatorname{Tr} u, \quad J_{5}=\operatorname{Tr}\left(u^{2}\right), \quad J_{6}=\operatorname{det} u .
\end{gathered}
$$

Explicitly, Eq. (3.3) looks as follows

$$
\begin{gathered}
R(w, E)=k_{6} E^{6}+k_{4} E^{4}+k_{2} E^{2}+\ell_{4} w E^{4}+\ell_{2} w^{2} E^{2}+m_{2} w E^{2} \\
+n_{3} w^{3}+n_{2} w^{2}+n_{1} w+n_{0}=0, \\
k_{6}=k^{2}, \quad k=I_{1} I_{2} I_{3}, \quad k_{4}=-k J_{1}, \quad \ell_{4}=-k \operatorname{Tr}(I), \\
k_{2}=J_{3}-J_{2} J_{4}+2^{-1} k\left(J_{4}^{2}-J_{5}\right) \operatorname{Tr}\left(I^{-1}\right), \quad \ell_{2}=k \operatorname{Tr}\left(I^{-1}\right), \\
\left.m_{2}=J_{2}-k \operatorname{Tr}\left(I^{-1}\right) J_{4}, \quad n_{3}=-1, \quad n_{2}=J_{4}, \quad n_{1}=\left(J_{5}-J_{4}^{2}\right) / 2, \quad n_{0}=J_{6} .5\right)
\end{gathered}
$$

As $E$ goes to infinity, Eqs. (3.3)-(3.5) have three roots, $w_{i}=B_{i} E^{2}\left(1+0|E|^{-1}\right)$. Therefore, the surface $\Gamma$ is a 3 -sheeted ramified covering of the complex $E$ line. The projective closure of the curve $\Gamma$ has a singular point with coordinates $x_{0}=w^{-1}=0, x_{1}=E w^{-1}=0$, where all three sheets of the surface $\Gamma$ are intersecting for $E \rightarrow \infty$. In order to resolve this singularity, we introduce new coordinates $z=E^{-1}, W=w E^{-2}$. In terms of these variables the equation in (3.5) acquires the form

$$
k_{6}+k_{4} z^{2}+k_{2} z^{4}+\ell_{4} W+\ell_{2} W^{2}+m_{2} W z^{2}+n_{3} W^{3}+n_{2} W^{2} z^{2}+n_{1} W z^{4}+n_{0} z^{6}=0 .
$$

This equation has three roots for $z=0$; the roots are $W_{i}=I_{1} I_{2} I_{3} I_{i}^{-1}=B_{i}$, and they determine three points $P_{1}^{0}, P_{2}^{0}, P_{3}^{0}$, which realize a nonsingular closure of the surface $\Gamma$ at infinity.

The surface $\Gamma$, as given by Eq. (3.5), is invariant under an involution $\sigma:(w, E)$ $\rightarrow(w,-E)$. Let us consider a map $f:(w, E) \rightarrow\left(w, E_{1}=E^{2}\right)$ of the surface $\Gamma$ onto a surface $\Gamma_{1}=\Gamma / \sigma$, that is given by the equation

$$
\begin{aligned}
R_{1}\left(w, E_{1}\right)= & k_{6} E_{1}^{3}+k_{4} E_{1}^{2}+k_{2} E_{1}+\ell_{4} w E_{1}^{2}+\ell_{2} w^{2} E_{1} \\
& +m_{2} w E_{1}+n_{3} w^{3}+n_{2} w^{2}+n_{1} w+n_{0}=0 .
\end{aligned}
$$

This is a third-order equation, so the genus of the surface $\Gamma_{1}$ is $g\left(\Gamma_{1}\right)=1$, and its Euler characteristic is $\chi\left(\Gamma_{1}\right)=0$, so $\Gamma_{1}=\mathbb{T}^{2}$. The genus of the Riemannian surface $\Gamma$ is calculated easily by means of the map $f$. The latter is a two-sheeted ramified covering, and it has 6 branch points, $f\left(P_{i}^{0}\right), f\left(Q_{i}\right), i=1,2,3$, where $Q_{i}$ are three 
roots (in the general case) of Eq. (3.5) for $E=0$. So we obtain, by means of the Riemann-Hurwitz formula,

$$
\chi(\Gamma)=2 \chi\left(\Gamma_{1}\right)-6=-6, \quad g(\Gamma)=1-\chi(\Gamma) / 2=4 .
$$

It is noteworthy that for the considered integrable dynamical system (2.8) the genus of the corresponding Riemannian surface is $g(\Gamma)=4$, that is higher than the dimension, $d=3$, of the invariant tori $\mathbb{T}^{3}$, where the quasi-periodical dynamics takes place, as is shown in Sect. 2.

2. For a complete description of rotation of a rigid body, it is sufficient to know the time dependence of components of the angular velocity vector, so the purpose of our work here is to get explicit formulae for the components $\omega_{i}^{j}(t)$. The method we are going to apply $[22,23,12-14]$ is to construct certain analytical (except at a finite number of points) functions in the Riemannian surface $\Gamma$ (functions of the Baker-Akhiezer type [22-24]). On one hand, these functions depend on parameters of the system given in Eqs. (2.8), (3.1); on the other hand, they are uniquely expressed in terms of the Riemann $\theta$-function for the surface $\Gamma$, in view of the uniqueness theorem. This relation enables one to get an expression for the components $\omega_{i}^{j}(t)$ via the Riemann $\theta$-function.

Fulfillment of Eq. (3.1) is equivalent to commutativity of the matrix operators $F=\partial / \partial t+Q$ and $L$. Let us consider the common eigenvector functions for these operators, which satisfy the conditions

$$
(\partial / \partial t+Q) \psi_{k}=0, \quad L \psi_{k}=w_{k} \psi_{k}, \quad R\left(w_{k}, E\right)=0, \quad k=1,2,3 .
$$

In combination, components of the vector functions $\psi_{k}$ are a $3 \times 3$ matrix with elements $\psi_{k}^{j}(t, E)$. Denote by $\varphi_{i}^{k}(t, E)$ elements of its inverse matrix $\varphi(t, E)$. Introduce the matrix-valued function $g\left(t, P_{k}\right)$ with the elements $g_{i}^{j}\left(t, P_{k}\right)$, which depend on a point $P_{k}=\left(w_{k}, E\right)$ in the Riemannian surface $\Gamma$ and time $t$; by definition,

$$
g_{i}^{j}\left(t, P_{k}\right)=\psi_{k}^{j}(t, E) \varphi_{i}^{k}(t, E)
$$

The matrices $\psi(t, E), \varphi(t, E), g\left(t, P_{k}\right)$ due to (3.8) satisfy the equations

$$
\partial \psi / \partial t=-Q \psi, \quad \partial \varphi / \partial t=\varphi Q, \quad \partial g / \partial t=[g, Q] .
$$

It is natural to consider three vector functions $\psi_{k}(t, E)$ as one vector function $\psi\left(t, P_{k}\right)$ with the components $\psi^{j}\left(t, P_{k}\right)$ depending on a point $P_{k}=\left(w_{k}, E\right)$ in the Riemannian surface $\Gamma: \psi^{j}\left(t, P_{k}\right)=\psi_{k}^{j}(t, E)$. Analogously, the vector function $\varphi\left(t, P_{k}\right)$ with the components $\varphi_{i}\left(t, P_{k}\right)=\varphi_{i}^{k}(t, E)$ is determined. Functions $\psi^{j}\left(t, P_{k}\right)$ are entire in the affine part of the Riemannian surface $\Gamma$, functions $\varphi_{i}\left(t, P_{k}\right)$ are meromorphic, their divisors in the affine part of $\Gamma$ have the form

$$
\left(\psi^{j}(t, P)\right)_{a}=d^{j}(t), \quad\left(\varphi_{i}(t, P)\right)_{a}=d_{i}(t)-D_{r},
$$

where $D_{r}$ is the divisor of the branch points of the Riemannian surface $\Gamma$ over the complex $E$-plane (it does not depend on time $t$ ). By the Riemann-Hurwitz formula we have $\chi(\Gamma)=3 \chi\left(S^{2}\right)-\operatorname{deg}\left(D_{r}\right)$. After substitution $\chi(\Gamma)=-6, \chi\left(S^{2}\right)=2$, we obtain $\operatorname{deg}\left(D_{r}\right)=12$. 
A consequence of the latter equation in (3.10), and of the definition $Q=\omega-E I$, is an asymptotic expansion for the matrix $g(t, P)$ at $P \rightarrow P_{k}^{0}\left(z=E^{-1} \rightarrow 0, W \rightarrow B_{k}\right)$

$$
\begin{gathered}
g=g_{0}+g_{1} z+g_{2} z^{2}+\ldots, \quad\left(g_{0}\right)_{i}^{j}=\delta_{k}^{j} \delta_{i}^{k}, \\
\left(g_{1}\right)_{i}^{j}=\left(I_{i}-I_{j}\right)^{-1}\left(\delta_{k}^{j} \omega_{i}^{k}-\delta_{i}^{k} \omega_{k}^{j}\right), \\
\left(I_{i}-I_{j}\right)\left(g_{2}\right)_{i}^{j}=-\left(\dot{g}_{1}\right)_{i}^{j}+\left(I_{s}-I_{i}\right)^{-1}\left(\delta_{k}^{j} \omega_{s}^{k} \omega_{i}^{s}-\omega_{k}^{j} \omega_{i}^{k}\right)+\left(I_{i}-I_{s}\right)^{-1}\left(\delta_{i}^{k} \omega_{s}^{j} \omega_{k}^{s}-\omega_{k}^{j} \omega_{i}^{k}\right)
\end{gathered}
$$

(summation over the index $s$ is implied, and there is no summation in $k$ ). The function $g_{i}^{j}(t, P)$ due to the definition (3.9) and the asymptotic expansion (3.12) is meromorphic in the whole Riemannian surface $\Gamma$, its divisor $\left(g_{i}^{j}(t, P)\right)$ looks as follows [14]

$$
\left(g_{i}^{j}(t, P)\right)=d_{i}(t)+d^{j}(t)-D_{r}+2\left(P_{1}^{0}+P_{2}^{0}+P_{3}^{0}\right)-P_{i}^{0}-P_{j}^{0} .
$$

For a meromorphic function $g_{i}^{j}(t, P)$ one has $\operatorname{deg}\left(\left(g_{i}^{j}(t, P)\right)\right)=0$, hence due to $\operatorname{deg}\left(D_{r}\right)=12$ from (3.13) it follows $\operatorname{deg} d^{j}(t)=\operatorname{deg} d_{i}(t)=g(\Gamma)=4$.

Introduce the functions

$$
\psi^{j}(t, \tau, P)=\psi^{j}(t, P) / \psi^{j}(\tau, P), \quad \psi_{i}(t, \tau, P)=\varphi_{i}(t, P) / \varphi_{i}(\tau, P) .
$$

The functions $\psi^{j}(t, \tau, P), \psi_{i}(t, \tau, P)$ are independent of normalization of solutions of Eqs. (3.8) and are meromorphic in the affine part of the Riemannian surface $\Gamma$; their divisors are of the form,

$$
\left(\psi^{j}(t, \tau, P)\right)_{a}=d^{j}(t)-d^{j}(\tau), \quad\left(\psi_{i}(t, \tau, P)\right)_{a}=d_{i}(t)-d_{i}(\tau) .
$$

Using the definitions (3.9)-(3.14) we obtain

$$
\partial \ln g_{i}^{j}(t, P) / \partial t=\partial \ln \psi^{j}(t, 0, P) / \partial t+\partial \ln \psi_{i}(t, 0, P) / \partial t .
$$

Hence we have, taking into account the asymptotics (3.12) for $P \rightarrow P_{j}^{0}$,

$$
\omega_{i}^{j}(t)=C_{i}^{j} \lim _{P \rightarrow P_{j}^{0}} \psi^{j}(t, 0, P) \psi_{i}(t, 0, P),
$$

where $C_{i}^{j}$ are constants.

Using the formulae (3.16) it is possible to express the angular velocities $\omega_{i}^{j}(t)$ in terms of the $\theta$-functions of the Riemannian surfaces $\Gamma$. For that it is necessary to determine analytical properties of the functions $\psi^{j}(t, \tau, P), \psi_{i}(t, \tau, P)$, due to which they are uniquely expressed in terms of the Riemannian $\theta$-functions. Let us prove that at three points $P_{k}^{0}$ the functions $\psi^{j}(t, \tau, P)$ and $\psi_{i}(t, \tau, P)$ have essential singularities with the asymptotics (for $z \rightarrow 0, P \rightarrow P_{k}^{0}$ )

$$
\begin{aligned}
& \psi^{j}(t, \tau, P)=\alpha^{j}(t, \tau, P) \exp \left((t-\tau) I_{k} z^{-1}\right), \\
& \psi_{i}(t, \tau, P)=\alpha_{i}(t, \tau, P) \exp \left(-(t-\tau) I_{k} z^{-1}\right),
\end{aligned}
$$

where $\alpha^{j}(t, \tau, P), \alpha_{i}(t, \tau, P)$ are functions meromorphic in a neighbourhood of the points $P_{k}^{0}$. The functions $\psi^{j}(t, \tau, P)$ and $\psi_{i}(t, \tau, P)$ due to (3.8)-(3.14) satisfy the 
following equations,

$$
\begin{aligned}
\partial \ln \psi^{j}(t, \tau, P) / \partial t & =\dot{\psi}^{j}(t, P) / \psi^{j}(t, P)=-Q_{s}^{j} \psi^{s}(t, P) / \psi^{j}(t, P) \\
& =z^{-1} I_{j}-\omega_{s}^{j}(t) \psi^{s}(t, P) / \psi^{j}(t, P) \\
& =z^{-1} I_{j}-\omega_{s}^{j}(t) g_{x}^{s}(t, P) / g_{x}^{j}(t, P) \\
\partial \ln \psi_{i}(t, \tau, P) / \partial t & =\dot{\varphi}_{i}(t, P) / \varphi_{i}(t, P)=Q_{i}^{s} \varphi_{s}(t, P) / \varphi_{i}(t, P) \\
& =-z^{-1} I_{i}+\omega_{i}^{s}(t) \varphi_{s}(t, P) / \varphi_{i}(t, P) \\
& =-z^{-1} I_{i}+\omega_{i}^{s}(t) g_{s}^{x}(t, P) / g_{i}^{x}(t, P)
\end{aligned}
$$

In the formulae (3.18) (which meanings are independent of the index $x$ ) we set $x=k$ and use the expansion (3.12); after that at $P \rightarrow P_{k}^{0}$ we obtain

$$
\begin{aligned}
& \partial \ln \psi^{j}(t, \tau, P) / \partial t=z^{-1} I_{k}+f_{j k}^{1}(t, z), \\
& \partial \ln \psi_{i}(t, \tau, P) / \partial t=-z^{-1} I_{k}+f_{i k}^{2}(t, z),
\end{aligned}
$$

where functions $f_{j k}^{1}(t, z), f_{i k}^{2}(t, z)$ are holomorphic in a neighbourhood of the point $P_{k}^{0}$. The asymptotics (3.17) follows now from the formulae (3.19) and the identities $\psi^{j}(t, t, P)=\psi_{i}(t, t, P)=1$. From the formulae (3.18) at $j=k=x$ due to $\omega_{j}^{j}=0$, it follows $f_{j j}^{1}\left(t, P_{j}^{0}\right)=0$, analogously $f_{i i}^{2}\left(t, P_{i}^{0}\right)=0$, hence from (3.19) we obtain

$$
\alpha^{j}\left(t, \tau, P_{j}^{0}\right)=1, \quad \alpha_{i}\left(t, \tau, P_{i}^{0}\right)=1 .
$$

The functions $\psi^{j}(t, \tau, P)$ and $\psi_{i}(t, \tau, P)$ are three-point functions of the BakerAkhiezer type, with essential singularities at the points $P_{k}^{0}$ and the pole divisors $d^{j}(\tau)$ and $d_{i}(\tau)$, respectively. The functions $\psi^{j}(t, \tau, P)$ and $\psi_{i}(t, \tau, P)$ at the normalizations (3.20) are determined uniquely (this is the uniqueness theorem) by means of the following constructions. Let $a_{1}, \ldots, a_{4}, b_{1}, \ldots, b_{4}$ be a basis of cycles in the Riemannian surface $\Gamma$, with the intersection indices $a_{i} \circ a_{j}=b_{i} \circ b_{j}=0$, $a_{i} \circ b_{j}=\delta_{i j} ; \omega_{1}, \omega_{2}, \omega_{3}, \omega_{4}$ are holomorphic differentials in $\Gamma$, with the normalization conditions $\oint_{a_{k}} \omega_{j}=2 \pi i \delta_{j}^{k}$. The elements of the Riemannian matrix $B(\Gamma)$ are defined by the formulas $B_{i j}=\oint_{b_{j}} \omega_{i}$. The classical Riemann $\theta$-function, corresponding to the surface $\Gamma$, is given by its Fourier series,

$$
\theta\left(z_{1}, z_{2}, z_{3}, z_{4}\right)=\sum_{\mathbf{N} \in Z^{4}} \exp \left(2^{-1}(B \mathbf{N}, \mathbf{N})+(\mathbf{N}, \mathbf{z})\right) .
$$

The Abel map $\mathbf{A}: \Gamma \rightarrow \mathrm{Jac} \Gamma$, where Jac $\Gamma$ is the Jacobi variety of the surface $\Gamma$ (Jac $\Gamma$ $\left.=\mathbb{T}^{8}=\mathbb{C}^{4} /\{2 \pi i \mathbf{N}+B \mathbf{M}\}\right)$ is given by the formulae $\mathbf{A}(P)=\left(A_{1}(P), \ldots, A_{4}(P)\right)$, where $A_{j}(P)=\int_{P_{j}}^{P} \omega_{j}$. For the divisor $D=c_{1} P_{1}+\ldots+c_{s} P_{s}$, the Abel map is $\mathbf{A}(D)$ $=c_{1} \mathbf{A}\left(P_{1}\right)+\ldots+c_{s} \mathbf{A}\left(P_{s}\right)$. Let $\Omega_{k} \quad(k=1,2,3)$ be the second-kind Abelian differential in $\Gamma$, the principal part of which at the point $P_{k}^{0}$ is $-d z / z^{2}$; the normalization conditions are $\oint_{a_{j}} \Omega_{k}=0, j=1, \ldots, 4$. Vectors $\mathbf{U}_{k}$ have components $U_{k}^{j}=\oint_{b_{j}} \Omega_{k}, j=1,2,3,4 ; k=1,2,3$. We introduce the notations $\Omega=I_{1} \Omega_{1}+I_{2} \Omega_{2}$ $+I_{3} \Omega_{3}$ and $\mathbf{U}=I_{1} \mathbf{U}_{1}+I_{2} \mathbf{U}_{2}+I_{3} \mathbf{U}_{3}$. The three-point Baker-Akhiezer functions 
$\psi^{j}$ and $\psi_{i}$ are defined by the formulae

$$
\begin{aligned}
& \psi^{j}(t, \tau, P)=c^{j} \exp \left((t-\tau) \int_{P_{0}}^{P} \Omega\right) \frac{\theta\left(\mathbf{A}(P)-\mathbf{A}\left(d^{j}(\tau)\right)+(t-\tau) \mathbf{U}-\mathbf{K}\right)}{\theta\left(\mathbf{A}(P)-\mathbf{A}\left(d^{j}(\tau)\right)-\mathbf{K}\right)}, \\
& \psi_{i}(t, \tau, P)=c_{i} \exp \left(-(t-\tau) \int_{P_{0}}^{P} \Omega\right) \frac{\theta\left(\mathbf{A}(P)-\mathbf{A}\left(d_{i}(\tau)\right)-(t-\tau) \mathbf{U}-\mathbf{K}\right)}{\theta\left(\mathbf{A}(P)-\mathbf{A}\left(d_{i}(\tau)\right)-\mathbf{K}\right)} .
\end{aligned}
$$

Here $\mathbf{K}$ is the vector of Riemann's constants; it has the components

$$
K_{j}=\pi i+\frac{1}{2} B_{j j}-\frac{1}{2 \pi i} \sum_{k \neq j} \oint_{a_{k}}\left(\omega_{k}(P) \int_{P_{0}}^{P} \omega_{j}\right), \quad j=1,2,3,4 .
$$

The factors $c^{j}$ and $c_{i}$ are determined by the normalization conditions (3.20), and have the form

$$
\begin{aligned}
& c^{j}=\exp \left((\tau-t) \xi^{j}\right) \frac{\theta\left(\mathbf{A}\left(P_{j}^{0}\right)-\mathbf{A}\left(d^{j}(\tau)\right)-\mathbf{K}\right)}{\theta\left(\mathbf{A}\left(P_{j}^{0}\right)-\mathbf{A}\left(d^{j}(\tau)\right)+(t-\tau) \mathbf{U}-\mathbf{K}\right)}, \\
& c_{i}=\exp \left(-(\tau-t) \xi^{i}\right) \frac{\theta\left(\mathbf{A}\left(P_{i}^{0}\right)-\mathbf{A}\left(d_{i}(\tau)\right)-\mathbf{K}\right)}{\theta\left(\mathbf{A}\left(P_{i}^{0}\right)-\mathbf{A}\left(d_{i}(\tau)\right)-(t-\tau) \mathbf{U}-\mathbf{K}\right)},
\end{aligned}
$$

where the constants $\xi^{\ell}(\ell=1,2,3)$ are defined as $\xi^{\ell}=I_{1} \xi_{1}^{\ell}+I_{2} \xi_{2}^{\ell}+I_{3} \xi_{3}^{\ell}$, and

$$
\xi_{k}^{\ell}=\left(1-\delta_{k}^{\ell}\right) \int_{P_{0}}^{P_{0}^{0}} \Omega_{k}+\delta_{k}^{\ell} \lim _{P \rightarrow P_{k}^{0}}\left(\int_{P_{0}}^{P} \Omega_{k}-z^{-1}\right) .
$$

Next we use the notations $\sigma f$ for the image of a function $f$ under the involution $\sigma$ : $(w, E) \rightarrow(w,-E)$ in the curve $\Gamma$, and $\sigma D$ is the image of the divisor $D$.

Lemma 1. The following equalities are valid in the Jacobi variety $\operatorname{Jac} \Gamma$,

$$
\begin{aligned}
& \mathbf{A}\left(d^{j}(t)+\sigma d^{j}(t)\right)=\mathbf{A}\left(d^{j}(\tau)+\sigma d^{j}(\tau)\right), \\
& \mathbf{A}\left(d_{i}(t)+\sigma d_{i}(t)\right)=\mathbf{A}\left(d_{i}(\tau)+\sigma d_{i}(\tau)\right) .
\end{aligned}
$$

Actually, one can consider the functions $\sigma \psi^{j}(t, \tau, P)$ and $\sigma \psi_{i}(t, \tau, P)$. By definition, $\sigma f(W, z)=f(W,-z), \sigma\left(P_{k}^{0}\right)=P_{k}^{0}$, so the asymptotics of the functions $\sigma \psi^{j}$ and $\sigma \psi_{i}$ as $z$ goes to zero, and $P \rightarrow P_{k}^{0}$, is the same as that in Eqs. (3.17); the only difference is the sign in the exponential factors. Consequently, the products $\psi^{j} \cdot \sigma \psi^{j}(t, \tau, P)$ and $\psi_{i} \cdot \sigma \psi_{i}(t, \tau, P)$ are meromorphic functions in $\Gamma$, and because of (3.15) their divisors are

$$
\begin{aligned}
& \left(\psi^{j} \cdot \sigma \psi^{j}(t, \tau, P)\right)=d^{j}(t)+\sigma d^{j}(t)-d^{j}(\tau)-\sigma d^{j}(\tau), \\
& \left(\psi_{i} \cdot \sigma \psi_{i}(t, \tau, P)\right)=d_{i}(\tau)+\sigma d_{i}(\tau)-d_{i}(t)-\sigma d_{i}(t) .
\end{aligned}
$$

Hence we get Eqs. (3.24), by Abel's theorem.

In the definition of Abel's map we choose a point $P_{0}$ in such a way that $\mathbf{A}\left(d^{1}(0)+\sigma d^{1}(0)\right)=0$, then we have, because of Eqs. (3.24),

$$
\mathbf{A}\left(d^{1}(t)+\sigma d^{1}(t)\right)=0
$$

Consequently, the vector $\mathbf{A}\left(d^{1}(t)\right)$ belongs to the Prym variety $\operatorname{Prym}_{\sigma} \Gamma \subset \mathrm{Jac} \Gamma$ (some properties and applications of Prym varieties have been discussed in 
$[17,19])$. The complex variety $\operatorname{Prym}_{\sigma} \Gamma$ is an abelian torus in $\mathrm{Jac} \Gamma$, its dimension, $\operatorname{dim}_{\mathbb{C}}\left(\operatorname{Prym}_{\sigma} \Gamma\right)=g(\Gamma)-g\left(\Gamma_{1}\right)=3$, is equal to the dimension of the invariant tori $\mathbb{T}^{3}$ characterizing the integrable system $(2.8)$ [the tori $\mathbb{T}^{3}$ are fixed by definite values of the integrals (3.4) that determine also the Riemannian surface $\Gamma]$. The constructions described above lead to the map $\Phi: \mathbb{T}^{3} \rightarrow \operatorname{Prym}_{\sigma} \Gamma \subset \operatorname{Jac} \Gamma$ :

$$
\boldsymbol{\Phi}\left(\omega_{i}^{j}(t), u_{i}^{j}(t)\right)=\mathbf{A}\left(d^{1}(t)\right) .
$$

As a result of this map, dynamics of the system (2.8) is linearized in the variety $\operatorname{Prym}_{\sigma} \Gamma$. Actually, because of (3.17), the divisor of zeroes of the function $\psi^{1}(t, 0, P)$ is $d^{1}(t)$. According to the explicit formula (3.22) and properties of the Riemann $\theta$-function, the image of the divisor $d^{1}(t)$ under the Abel map is

$$
\mathbf{A}\left(d^{1}(t)\right)=\mathbf{A}\left(d^{1}(0)\right)-t \mathbf{U} .
$$

A consequence of this equality is, in particular, that the vector $\mathbf{U}$ belongs to $\operatorname{Prym}_{\sigma} \Gamma$, and in general the trajectory $t \mathbf{U}$ fills densely everywhere the threedimensional (real) torus $\mathbb{T}_{1}^{3}$ in the Prym variety $\operatorname{Prym}_{\sigma} \Gamma$. Thus we have proven the following:

Theorem 2. Dynamics of the system (2.8) is linearized in the Prym variety $\operatorname{Prym}_{\sigma} \Gamma$ $C \mathrm{Jac} \Gamma$ by means of the map (3.26).

The vectors $\mathbf{A}\left(d_{i}(t)\right), \mathbf{A}\left(d^{j}(t)\right)$ are determined uniquely by the vectors $\mathbf{A}\left(d^{1}(t)\right)$ [14]. Actually, as the function $g_{i}^{j}(t, P)$ is meromorphic, we have $\mathbf{A}\left(\left(g_{i}^{j}(t, P)\right)\right)=0$ because of Abel's theorem. So we get from Eqs. (3.13)

$$
\begin{aligned}
& \mathbf{A}\left(d_{i}(t)\right)=\mathbf{A}\left(D_{r}-P_{1}^{0}-2 P_{2}^{0}-2 P_{3}^{0}+P_{i}^{0}-d^{1}(t)\right), \\
& \mathbf{A}\left(d^{j}(t)\right)=\mathbf{A}\left(P_{j}^{0}-P_{1}^{0}+d^{1}(t)\right) .
\end{aligned}
$$

Because of Eqs. (3.28) and (3.27), we have $\mathbf{A}\left(d_{i}(t)\right)-\mathbf{A}\left(d_{i}(0)\right)=t \mathbf{U}$ and $\mathbf{A}\left(d^{j}(t)\right)$ $-\mathbf{A}\left(d^{j}(0)\right)=-t \mathbf{U}$, so for different $i, j$ we get identical windings of the Prym variety $\operatorname{Prym}_{\sigma} \Gamma$.

In order to get explicit expressions for $\omega_{i}^{j}(t)$ we find the product of Eqs. (3.22) and (3.23); the result is

$$
\begin{aligned}
& \lim _{P \rightarrow P_{j}^{0}} \psi^{j}(t, 0, P) \psi_{i}(0, t, P)=\exp \left(t\left(\xi^{i}-\xi^{j}\right)\right) \\
& \quad \cdot \frac{\theta\left(\mathbf{A}\left(P_{j}^{0}\right)-\mathbf{A}\left(d_{i}(0)\right)-t \mathbf{U}-\mathbf{K}\right) \cdot \theta\left(\mathbf{A}\left(P_{i}^{0}\right)-\mathbf{A}\left(d_{i}(0)\right)-\mathbf{K}\right)}{\theta\left(\mathbf{A}\left(P_{i}^{0}\right)-\mathbf{A}\left(d_{i}(0)\right)-t \mathbf{U}-\mathbf{K}\right) \cdot \theta\left(\mathbf{A}\left(P_{j}^{0}\right)-\mathbf{A}\left(d_{i}(0)\right)-\mathbf{K}\right)} .
\end{aligned}
$$

Using the parity of the Riemann $\theta$-function and Eqs. (3.28) for $t=0$ we get finally, substituting (3.29) in Eqs. (3.16)

$$
\begin{aligned}
\omega_{i}^{j}(t) & =A_{i}^{j} \exp \left(t\left(\xi^{i}-\xi^{j}\right)\right) \frac{\theta\left(\mathbf{A}\left(P_{i}^{0}\right)-\mathbf{A}\left(P_{j}^{0}\right)+t \mathbf{U}+\mathbf{z}_{0}\right)}{\theta\left(t \mathbf{U}+\mathbf{z}_{0}\right)}, \\
\mathbf{z}_{0} & =\mathbf{A}\left(D_{r}-d^{1}(0)-P_{1}^{0}-2 P_{2}^{0}-2 P_{3}^{0}\right)+\mathbf{K} .
\end{aligned}
$$

This is the representation of the solutions of system (2.8) in terms of the Riemann $\theta$-function for the surface $\Gamma$, which is determined in Eqs. (3.3)-(3.5). Initial values of the solution sought for, $\omega_{i}^{j}(0), u_{i}^{j}(0)$, determine uniquely the Riemannian 
surface $\Gamma$ and all the analytical constructions described above, in particular, the divisor $d^{1}(0)$ and a vector $\mathbf{z}_{0}$. With these data at hand, the coefficients $A_{i}^{j}$ are determined from Eqs. (3.30) at $t=0$. Thus Eqs. (3.30) determine the time dependence of the angular velocities $\omega_{i}^{j}(t)$ from the initial data $\omega_{i}^{j}(0), u_{i}^{j}(0)$, and the rotation of the rigid body is described completely.

In view of Eqs. (3.30), the functions $\omega_{i}^{j}(t)$ are meromorphic in the whole complex $t$-plane. The functions $u_{i}^{j}(t)$, which are determined easily from $\omega_{k}^{l}(t)$ in the Eqs. (2.8), are also meromorphic.

\section{Integrability of an $n$-Dimensional Analog of the Rotation Problem for a Rigid Body in a Quadratic Potential Field}

Let us consider an $n$-dimensional analog of system (2.8),

$$
\dot{M}=[M, \omega]-[u, A], \quad \dot{u}=[u, \omega],
$$

where $M, \omega, u, A$ are $n \times n$ matrices, and $M^{t}=-M, \omega^{t}=-\omega, u^{t}=u, A^{t}=A$, $M_{i j}=c_{i j} \omega_{i j}, c_{i j}=c_{j i}$. Suppose $A$ is a diagonal matrix, $A_{i j}=a_{i} \delta_{i j}$, and there is a diagonal matrix $B, B_{i j}=b_{i} \delta_{i j}$, enabling one to relate $M$ to $\omega$ by the conditions [15]

$$
M_{i j}=\left(b_{i}-b_{j}\right)\left(a_{j}-a_{i}\right)^{-1} \omega_{i j} .
$$

In this case, Eq. (4.1) are equivalent to the following matrix equation depending on the spectral parameter $E$,

$$
\dot{L}=[L, Q], \quad L=B E^{2}+M E+u, \quad Q=\omega-A E .
$$

Equations (4.1) are Euler equations in a space conjugate to the Lie algebra $L_{n^{2}}$, elements of which are matrices that can be represented in the form $\ell=M+u$, and the commutators are given in (2.9). Orbits of action of the corresponding Lie group $G_{n^{2}}$ in the space $L_{n^{2}}^{*}$ are symplectic manifolds the dimension of which is $n^{2}-n$; they are determined by $n$ conditions $\lambda_{1}(u)=c_{1}, \ldots, \lambda_{n}(u)=c_{n}$, where $\lambda_{i}(u)$ are eigenvalues of the matrix $u$. In orbits $\mathcal{O}$, system (4.1) is Hamiltonian; its Hamiltonian is $H=\operatorname{Tr}\left(\frac{1}{2} M \omega-A u\right)$. Under conditions (4.2), system (4.1) is completely integrable in $\Gamma$, in Liouville's sense. The integrals of this system, which are in involution, are coefficients of the equation

$$
R(w, E)=\operatorname{det}\left(B E^{2}+M E+u-w \cdot 1\right)=0 .
$$

Let us calculate genus $g(\Gamma)$ for the Riemannian surface $\Gamma$ that is determined by Eq. (4.3) (the singular point for infinite $|E|$ is resolved in the coordinates $z=E^{-1}$, $\left.W=w E^{-2}\right)$. In view of the definition $M^{t}=-M, u^{t}=u$, we have

$$
R(w, E)=\operatorname{det}(L-w \cdot 1)=\operatorname{det}\left(L^{t}-w \cdot 1\right)=R(w,-E) .
$$

Therefore, only even powers of $E$ are present in Eq. (4.3), and the involution $\sigma: \sigma(w, E)=(w,-E)$ is acting in the surface $\Gamma$. Introduce the notation $E_{1}=E^{2}$, and let $\Gamma_{1}=\Gamma / \sigma$ be the Riemannian surface which is fixed by the equation $R\left(w, E_{1}^{1 / 2}\right)=0$. The surface $\Gamma_{1}$ is of degree $n$, it has no singularities at infinity for $b_{i} \neq b_{j}$, so its genus is $g\left(\Gamma_{1}\right)=\frac{1}{2}(n-1)(n-2)$ [25]. The mapping $f: \Gamma \rightarrow \Gamma_{1}, f(w, E)$ $=\left(w, E_{1}=E^{2}\right)$ is a ramified two-sheeted covering; in general it has $2 n$ branch 
points: $n$ points for $E=0$ and $n$ points for $z=E^{-1}=0$. So we get, using the Riemann-Hurwitz formula, the Euler characteristic of the surface $\Gamma-\chi(\Gamma)$ and its genus $g(\Gamma)$,

$$
\chi(\Gamma)=2 \chi\left(\Gamma_{1}\right)-2 n, \quad g(\Gamma)=2 g\left(\Gamma_{1}\right)+n-1=(n-1)^{2} .
$$

System (4.1) can be integrated in terms of the Riemannian $\theta$-functions for the surfaces $\Gamma$, given in (4.3); the construction is like that in Sect. 3. The analytical presentation of the resulting functions $\omega_{i}^{j}(t)$ has the same form as in Eqs. (3.30). Lemma 1 and Theorem 2 of Sect. 3 are also valid in the $n$-dimensional case; dynamics of system (4.1) is linearized in the Prym variety Prym $\sigma \Gamma \mathcal{J a c} \Gamma$. The complex dimension of the variety $\operatorname{Prym}_{\sigma} \Gamma$ equals the real dimension of the invariant tori for system (4.1),

$$
\operatorname{dim}_{\mathbb{C}}\left(\operatorname{Prym}_{\sigma} \Gamma\right)=g(\Gamma)-g\left(\Gamma_{1}\right)=\frac{1}{2} n(n-1)=\operatorname{dim}_{\mathbb{R}} \mathcal{O} / 2=\operatorname{dim}_{\mathbb{R}} T .
$$

In view of the explicit formulae of the form (3.30), solutions of the system (4.1) are meromorphic functions in the whole complex $t$ plane.

\section{Integrable Cases of the Problem}

\section{of Rigid Body Rotations in Nonlinear Newtonian Fields}

Let us consider a rigid body with a fixed point under the action of a force field with potential of the form

$$
\varphi=\frac{1}{2} \sum_{\alpha, \beta=1}^{3} a_{\alpha \beta}(|\mathbf{x}|) x^{\alpha} x^{\beta}, \quad|\mathbf{x}|=\left(\left(x^{1}\right)^{2}+\left(x^{2}\right)^{2}+\left(x^{3}\right)^{2}\right)^{1 / 2},
$$

where $a_{\alpha \beta}(|\mathbf{x}|)$ are arbitrary differentiable functions of variable $|\mathbf{x}|$. Newtonian potentials of this form which satisfy the Laplace equation $\Delta \varphi=0$, are given by the formulae

$$
\begin{gathered}
\varphi=|\mathbf{x}|^{-5} \sum_{\alpha, \beta=1}^{3} c_{\alpha \beta} x^{\alpha} x^{\beta}+\sum_{\alpha, \beta=1}^{3} b_{\alpha \beta} x^{\alpha} x^{\beta}+c|\mathbf{x}|^{-1}, \\
c_{11}+c_{22}+c_{33}=0, \quad b_{11}+b_{22}+b_{33}=0
\end{gathered}
$$

Next we introduce a fourth-rank tensor, which is symmetrical in two pairs of indices $\alpha, \beta$ and $i, k$ and is a generalization of the inertia tensor $I_{i k}$, as given in Eq. (2.3),

$$
T_{\alpha \beta i k}=\int_{T} \varrho(\mathbf{r}) a_{\alpha \beta}(|\mathbf{r}|)\left(\delta_{i k} \sum_{\ell=1}^{3}\left(r^{\ell}\right)^{2}-r^{i} r^{k}\right) d r^{1} d r^{2} d r^{3} .
$$

Theorem 3. If the tensor $T_{\alpha \beta i k}$ can be represented as

$$
T_{\alpha \beta i k}=A_{\alpha \beta} I_{i k}+B_{\alpha \beta} \delta_{i k}+\delta_{\alpha \beta} C_{i k},
$$

where $A, B, C$ are arbitrary symmetrical matrices, rotation of the rigid body with a fixed point $O\left(x^{i}=0\right)$ in the force field with a potential (5.1) is completely integrable in Liouville's sense. The solutions are expressed in terms of $\theta$-functions for Riemannian surfaces. 
If $a_{\alpha \beta}(|\mathbf{x}|)=$ const, conditions (5.3) hold evidently, so Theorem 3 is a generalization of Theorem 1 . If the rigid body is spherical, and its density is $\varrho(\mathbf{r})$ $=\varrho_{1}(\mathbf{r} /|\mathbf{r}|) \varrho_{2}(|\mathbf{r}|)$, conditions (5.3) are satisfied for arbitrary functions $a_{\alpha \beta}(|\mathbf{x}|)$ (with $B_{\alpha \beta}=C_{i k}=0$ ), so rotation of the rigid body in a force field with an arbitrary potential (5.1) is completely integrable.

Let $Q(t)$ be an orthogonal matrix determining the transformation from the Lagrange coordinates $r^{k}$, which are referred to the $S$ frame, to the Euler coordinates $x^{i}$, namely $x^{i}=\sum_{k=1}^{3} Q_{k}^{i}(t) r^{k}$. By definition, $\dot{Q}=Q \omega$, where $\omega$ is the angular velocity matrix. In the reference frame $S$, potential of the problem, (5.1), is written down as

$$
\varphi=2^{-1} \sum_{\alpha, \beta, n, \ell=1}^{3} a_{\alpha \beta}(|\mathbf{r}|) Q_{n}^{\alpha} Q_{\ell}^{\beta} r^{n} r^{\ell} .
$$

Components of the torque acting to the rigid body in the force field with potential $\varphi$, as given in Eq. (5.1), are determined from the expressions

$$
K_{i}=\int_{T}\left(\mathbf{r} \times \varrho \frac{\partial \varphi}{\partial \mathbf{r}}\right)_{i} d r^{1} d r^{2} d r^{3}=\int_{T} \varepsilon_{i m n} r^{m} \varrho(\mathbf{r}) a_{\alpha \beta}(|\mathbf{r}|) Q_{n}^{\alpha} Q_{\ell}^{\beta} r^{\ell} d r^{1} d r^{2} d r^{3}
$$

(everywhere summation over repeated indices is implied). A skew-symmetric matrix $K$, corresponding to the torque (5.5) by the isomorphism of (2.6), has the following components

$$
K_{j k}=-\varepsilon_{i j k} K_{i}=T_{\alpha \beta j \ell} Q_{k}^{\alpha} Q_{\ell}^{\beta}-T_{\alpha \beta k \ell} Q_{j}^{\alpha} Q_{\ell}^{\beta} .
$$

Putting expressions (5.3) into (5.6) we get

$$
K_{j k}=I_{j \ell} A_{\alpha \beta} Q_{k}^{\alpha} Q_{\ell}^{\beta}-I_{k \ell} A_{\alpha \beta} Q_{j}^{\alpha} Q_{\ell}^{\beta} .
$$

We introduce another matrix $u=Q^{t} A Q$. Equation (5.7) written in the matrix form is $K=I u-u I=-[u, I]$. So equations determining the time dependences of the angular momentum matrix, and the matrix $u$, are

$$
\dot{M}=[M, \omega]-[u, I], \quad \dot{u}=[u, \omega],
$$

since $\dot{Q}=Q \omega$. Equations (5.8) determine completely rotation of a rigid body in the force field with potential (5.1), under conditions (5.3). Evidently, these equations coincide with those in (2.8), so the statement of Theorem 3 is a consequence of results presented in Sects. 2 and 3.

\section{References}

1. Euler, L.: Decouverte d'une nouveau principe de mechanique. Memoires de l'Acad. des Sci. de Berlin 14, 154-193 (1758)

2. Lagrange, J.: Mechanique analytique. Paris: Gauthier-Villars 1888

3. Kowalewski, S.: Sur le probleme della rotation d'un corps solide autour d'un point fixe. Acta Math. 12, 177-232 (1889)

4. Brun, F.: Rotation kring fix punkt. Öfversigt at Kongl. Svenska Vetenskaps Akademies Förhadlingar, Stockholm 7, 455-468 (1893)

5. Brun, F.: Rotation kring fix punkt II, III. Ark. Mat. Ast. Fys. 4, 4, 1-4 (1907); 6, 5, 1-10 (1909) 
6. Goryachev, D.I.: Some general integrals in the problem of dynamics of a rigid body (in Russian). Warsaw: Warsaw Educational District Printery 1910

7. Clebsch, A.: Über die Bewegung eines Körpers in einer Flüssigkeit. Math. Ann. 3, 238-262 (1871)

8. Steklov, V.A.: On motion of a rigid body in fluid (in Russian). Kharkov: Darre Printing House 1893

9. Lyapunov, A.M.: A new case of integrability for differential equations describing motion of a rigid body in fluid (in Russian). Trans. Kharkov Math. Soc. 4, 3-7 (1893)

10. Chaplygin, S.A.: A new particular solution for the problem of motion of a rigid body in fluid (in Russian). Proc. of Physical Section of the Russian Imper. Soc. of Naturalists 11, 101-110 (1902)

11. Jacobi, C.G.J.: Vorlesungen über Dynamik, Königsberg, 1866

12. Novikov, S.P.: A periodical problem for the Korteweg-de Vries equation. Funct. Anal. Appl. 8, 54-66 (1974)

13. Dubrovin, B.A., Matveev, V.B., Novikov, S.P.: Nonlinear equations of the Korteweg-de Vries type, finite-zone linear operators and abelian manifolds. Usp. Mat. Nauk 31, 55-136 (1976)

14. Dubrovin, B.A.: Completely integrable Hamiltonian systems related to matrix operators, and abelian manifolds. Funct. Anal. Appl. 11, 4, 28-41 (1977)

15. Manakov, S.V.: A remark on integration of Euler dynamical equations for $n$-dimensional rigid body. Funct. Anal. Appl. 10, 93-94 (1976)

16. Adler, M., van Moerbeke, P.: Completely integrable systems, Euclidean Lie algebras, and curves. Adv. Math. 38, 267-317 (1980)

17. Adler, M., van Moerbeke, P.: Linearization of Hamiltonian systems, Jacobi varieties, and representation theory. Adv. Math. 38, 318-379 (1980)

18. Perelomov, A.M.: A few comments on integrability of equations of motion of rigid body in a fluid. Funct. Anal. Appl. 15, 83-86 (1981)

19. Haine, L.: Geodesic flow on SO (4) and abelian surfaces. Math. Ann. 263, 435-472 (1983)

20. Arnold, V.I.: Mathematical methods of classical mechanics. Moscow: Nauka 1974

21. Novikov, S.P., Shmeltser, I.: Periodical solutions of the Kirchhoff equations for free motion of a rigid body in fluid, and the extended Lyusternik-Schnirelman-Morse theory. I. Funct. Anal. Appl. 15, 54-66 (1981)

22. Baker, H.F.: Note on the foregoing paper "Commutative ordinary differential operators. J. L. Burchnall and T. W. Chaundy. Proc. Royal Soc. London A 118, 584-593 (1928)

23. Akhiezer, N.I.: Continual analog of orthogonal polynomials in a system of intervals. Dokl. Akad. Nauk SSSR 141, 2, 263-266 (1961)

24. Dubrovin, B.A.: Theta-functions and nonlinear equations. Usp. Mat. Nauk 36, 11-81 (1981)

25. Griffiths, P., Harris, J.: Principles of algebraic geometry. New York: Wiley 1978

Communicated by Ya. G. Sinai

Received February 10, 1984 
